\title{
A Perspective on Scintillation
}

\author{
Mark A. Walker \\ Special Research Centre for Theoretical Astrophysics, School of Physics A28, \\ University of Sydney, NSW 2006, Australia
}

Abstract. Some comments on interstellar scintillation are presented.

Quite apart from learning about the structure of the scattering medium, there are situations where radio-wave scattering gives information on source physics that would be very hard to obtain in any other way. A good example of this is the use of the interstellar scattering disk as a giant "telescope aperture" which can resolve the emission region of pulsars (Gwinn et al., these Proceedings, p. 327; Wolszczan \& Cordes 1987). However, there are also circumstances where we wish to avoid the influence of the interstellar medium and typically this drives us to work at high radio frequencies, since scintillation is known to be a low frequency phenomenon. But what does this statement mean? indeed is it even correct? and if so how high is high enough?

There are some excellent reviews of interstellar scintillation in the literature, for example Rickett (1990) and Narayan (1992), in which many aspects of scattering are described. In particular, Narayan shows graphically that the broad-band flux variations exhibit a peak at the transition between "strong" and "weak" scattering regimes. (Precisely: this statement holds for point-like sources and assumes-with good justification - a Kolmogorov spectrum of turbulence in the interstellar medium.) Physically this peak corresponds to substantial wavefront corrugations occurring on the same scale as the first Fresnel zone-it is not just the magnitude of the phase changes, but also their transverse length scale that determines the level of flux modulation. Moreover one can infer from existing models of the interstellar medium (e.g., Taylor \& Cordes 1993) that the transition frequency lies at several $\mathrm{GHz}$ for the majority of the extragalactic sky, and is even higher for distant ( $>1 \mathrm{kpc}$ ) objects in the Galactic plane. Thus, in respect of the flux modulations, at least, it is not right to think of scintillation as a low frequency phenomenon, and perhaps more correct to think of it as a high (radio) frequency phenomenon! (Other quantities, notably the angular size of the scattering disk, are monotonically increasing with wavelength.)

We tend to think of scintillation as being important at low frequencies partly because the radio astronomical data in which it is manifest are mostly low frequency $(<1 \mathrm{GHz})$ data. This can be understood as follows. Scintillation is particularly important for pulsars-because of their small angular size-and pulsars have very steep spectra, so are difficult to study at high frequencies. By contrast, sources with flatter spectra are typically radio galaxies or quasars which are relatively large and hence show much reduced levels of modulation (because there are many, independent scintillating "patches" across the source). In consequence the quantity of data on sources exhibiting deep scintillations, in the transition region and the "weak" scattering regime, is relatively small.

The source which has highlighted this circumstance is the recently discovered intra-hour variable quasar PKS 0405-385 (Kedziora-Chudczer et al., these Proceedings, p. 267). This source is sufficiently compact that it happily scintillates throughout the range from 1 to $22 \mathrm{GHz}$-implying a brightness temperature 
$\mathrm{T}_{\mathrm{b}} \sim 5 \times 10^{14} \mathrm{~K}$, well above the static inverse-Compton limit. While PKS 0405385 is the only quasar known to behave in this way, there is no reason to suppose that it will remain unique.

We can gain further insight into the old perspective on scintillation by noting the high brightness temperature required for a source to scintillate at frequencies of several $\mathrm{GHz}$-see Figure 1. The figure shows the characteristic angular size for radio sources of $T_{b}=10^{12}, 10^{15} \mathrm{~K}$, together with representative angular sizes associated with interstellar scattering for extragalactic sources at low/high Galactic latitude. From this figure we can immediately see that most synchrotron sources (for which $T_{b}<10^{12} \mathrm{~K}$ ) are sufficiently large that they will not scintillate except at low frequencies and low Galactic latitudes, where the scattering disk is larger than the angular size of the source. By contrast, we also see that a source having $T_{b} \sim 10^{15} \mathrm{~K}$ will exhibit broad-band scintillations at essentially all radio frequencies, irrespective of its Galactic latitude. For a synchrotron source, such a high brightness temperature would require either ultra-relativistic motion with Lorentz factor $\Gamma \sim 10^{3}$ (thanks to Alan Marscher for pointing this out to me; see Wagner, these Proceedings, p. 257), or perhaps a transient "violation" of the inverse-Compton limit.

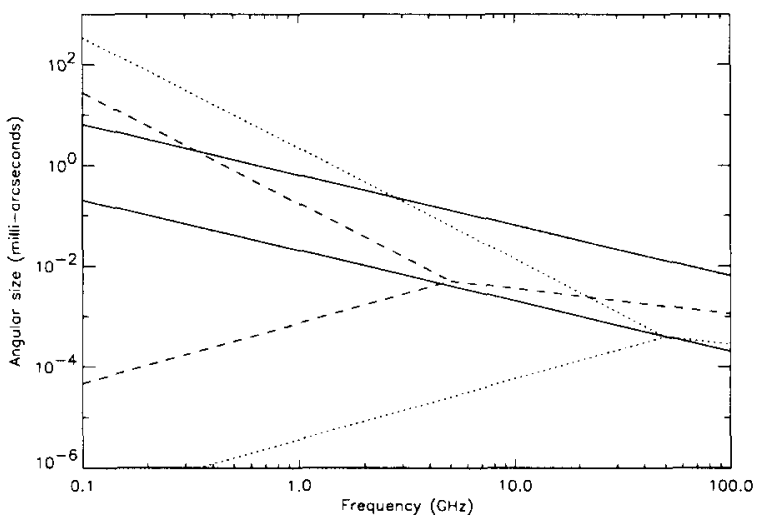

Figure 1. Angular sizes of $1 \mathrm{Jy}$ flat-spectrum sources-upper and lower solid lines corresponding to $\mathrm{T}_{\mathrm{b}}=10^{12}, 10^{15} \mathrm{~K}$, respectively-in comparison with the characteristic angular scales of interstellar scattering at high Galactic latitude (dashed lines), and at small latitude and modest longitude (dotted lines). Where the scattering is strong, there are two angular scales, with the larger one being associated with broad-band ("refractive") scintillation, and the smaller corresponding to narrow-band ("diffractive") scintillation. Extremely high brightness temperatures $\left(\mathrm{T}_{\mathrm{b}}>10^{15} \mathrm{~K}\right)$ are required in order to observe diffractive (interference) phenomena.

\section{References}

Narayan, R. 1992. Phil. Trans. R. Soc., 341, 151-165.

Rickett, B. J. 1990. ARA \&A, 28, 561-605.

Taylor, J. H., \& Cordes, J. M. 1993. ApJ, 411, 674-684.

Wolszczan, A., \& Cordes, J. M. 1987. ApJ, 320, L35-39. 